\title{
Potential benefit of (-)-epigallocatechin-3-gallate for macrovascular complications in diabetes
}

\author{
L. Tang ${ }^{1,2,3}$, L. $\mathrm{Li}^{1,2,3}$, J. Yang ${ }^{1,2,3}$ and C. Zeng ${ }^{1,2,3}$ \\ ${ }^{1}$ Department of Cardiology, Daping Hospital, Third Military Medical University, Chongqing, China \\ ${ }^{2}$ Chongqing Institute of Cardiology, Daping Hospital, Third Military Medical University, Chongqing, China \\ ${ }^{3}$ Chongqing Cardiovascular Clinical Research Center, Daping Hospital, Third Military Medical University, Chongqing, China
}

\begin{abstract}
Vascular problems are the most common complications in diabetes. Substantial evidence from epidemiological and pathophysiological studies show that hyperglycemia is a major risk factor for macrovascular complications in patients with diabetes. (-)-Epigallocatechin-3-gallate (EGCG), the major catechin derived from green tea, is known to exert a variety of cardiovascular beneficial effects. The protective effects of EGCG in diabetes are also evident. However, whether EGCG is beneficial against macrovascular complications that occur in diabetes remains unknown. Our previous studies demonstrated that treatment of EGCG inhibits high glucose-induced vascular smooth muscle cell proliferation and suppresses high glucose-mediated vascular inflammation in human umbilical vein endothelial cells. Therefore, we hypothesize that EGCG might be an effective potential candidate to reduce the macrovascular complications in diabetes.
\end{abstract}

Key words: (-)-Epigallocatechin-3-gallate; Diabetes; Macrovascular complications; Diabetes complications; Hypothesis

\section{Introduction}

The prevalence of diabetes has increased dramatically worldwide. Data from the Centers for Disease Control and Prevention show that nearly 26 million people in the United States have diabetes mellitus, with the vast majority (90-95\%) having type 2 diabetes mellitus (1). Diabetes is associated with increased incidence of macrovascular disease, particularly cardiovascular disease (CVD), including coronary heart disease, stroke and peripheral vascular disease, which is the major cause of morbidity and mortality in people with diabetes $(2,3)$. Substantial evidence has indicated that hyperglycemia is an important independent risk factor in the development and progression of diabetic macrovascular disease in patients with diabetes. Epidemiological studies also suggest that in people with type 2 diabetes, cardiovascular mortality is related to the degree of hyperglycemia (4), which was confirmed by another metaanalysis (5).

Both in vivo and in vitro studies have confirmed the contribution of high glucose to vascular injury. In cultured vascular smooth muscle cells (VSMCs), high glucose induced proliferation and oxidative injury $(6,7)$ and inhibited apoptosis (8). In cultured endothelial cells, high glucose induced apoptosis, up-regulated vascular cell adhesion and molecule-mediated adhesiveness, and increased permeability (9-11). In animal models, high-glucose induced neointimal formation in a carotid arterial balloon injury model (7), impaired endothelium-dependent vasorelaxation (12), and enhanced the production of vascular cell adhesion molecules (13). All the above studies show that hyperglycemia is an important risk factor for diabetic macrovascular disease. Therefore, inhibition of high glucose-induced abnormal vascular injury is an important issue.

\section{Beneficial effects of (-)-epigallocatechin- 3-gallate on cardiovascular diseases}

In recent years, phytochemicals have offered promising new options for the development of more effective therapeutic strategies for cardiovascular diseases and diabetes. Teas produced from the leaves of the plant Camellia sinensis are widely consumed beverages throughout the world. Among the consumed teas, green tea is the best studied for its health benefits. Epidemiological studies have suggested that consumption of green tea might prevent the incidence of various cardiovascular diseases, including atherosclerosis (14). Green tea contains many biologically active polyphenolic flavonoids, commonly known as tea polyphenols (15). The major components of tea polyphenols are the catechins,

Correspondence: C. Zeng: <chunyuzeng01@163.com> | J. Yang: <yangjianac@163.com> 
a family that includes (-)-epicatechin, (-)-epigallocatechin, (-)-epicatechin-3-gallate, and (-)-epigallocatechin-3-gallate (EGCG).

As the principal constituent, EGCG is a major polyphenolic constituent present in green tea and has been known to exert a variety of cardiovascular beneficial effects $(16,17)$. In vitro studies show that treatment with EGCG inhibited invasive activity of cultured human VSMC, restrained rat VSMC adhesion and migration, and repressed the angiotensin II-stimulated VSMC proliferation and adhesion molecule expression in human umbilical vein endothelial cells (18-21). The results in cultured cells were confirmed in animal studies. In the apolipoprotein E-null mice, EGCG treatment resulted in increased antioxidant capacity in local vascular tissue and systemic circulation, and reduced cuff-induced atherosclerotic plaque size (22). In spontaneously hypertensive rats, EGCG improved endothelial function, reduced systolic blood pressure, and attenuated myocardial ischemiareperfusion injury (23).

\section{Beneficial effects of EGCG on diabetes}

In recent years, several studies have investigated the potent anti-diabetic properties of EGCG. In diabetic animal models, EGCG delayed the onset of type 1 diabetes in spontaneous non-obese mice, reduced the increase of blood glucose levels and ameliorated the decrease of islet mass induced by low-doses of streptozotocin $(24,25)$, and ameliorated vascular reactivity in rats and enhanced glucose tolerance in rodents $(26,27)$. In vitro studies showed that treatment with EGCG improved the survival rate of isolated islets and reduced the loss of functional islet mass (28), and attenuated high glucose-induced harmful effects such as the down-regulation of the cardiac gap junction, insulin signaling blockade, embryonic vasculopathy and the expression of pro-inflammatory cytokines (29-32). Furthermore, EGCG is also beneficial against complications of diabetes. Yamabe et al. (33) found in a diabetic nephropathy rat model that EGCG ameliorated glucose toxicity and renal injury, thus alleviating renal damage caused by abnormal glucose metabolism-associated oxidative stress involved in renal lesions of diabetic nephropathy (Table 1).

\section{Potential benefit of EGCG on macrovascular complications}

A follow-up study showed the safety of EGCG in healthy individuals taking green tea polyphenol products in amounts equivalent to the EGCG content in 8-16 cups of green tea (800 mg EGCG) once a day or in divided doses twice a day for 4 weeks (34). There was a $>60 \%$ increase in the systemic availability of free EGCG. This result was in accordance with other studies, which reported that the long history of green tea consumption seems to have no obvious adverse effects $(35,36)$. Therefore, it might be possible that the extended use of green tea or EGCG by humans could build sufficient EGCG concentration in the plasma.

We propose the hypothesis that oral administration of EGCG might be a safe and effective therapeutic option to prevent/cure macrovascular complications in patients with diabetes. To test this hypothesis, we propose a systematic use of EGCG or green tea polyphenol mixture in patients with diabetes. Of course, the safety of EGCG should be verified in a diabetic animal model first. This should include genotoxic, acute and short-term toxicity, teratogenicity and reproductive toxicity (37-39), before attempting a large scale study involving human subjects.

In preliminary results of our investigations, we found that treatment with EGCG inhibited high glucose-induced VSMC proliferation by inhibiting PKC and ERK1/2 signaling in rat VSMCs (40) and suppressed high glucoseinduced vascular inflammation in human umbilical vein endothelial cells by inhibition of PKC and NF- $\mathrm{KB}$ signaling pathway (J. Yang, Y. Han, C. Chen, D. He, L. Zhou and

Table 1. Biological beneficial actions of (-)-Epigallocatechin-3-gallate on diabetes and cardiovascular diseases.

\begin{tabular}{cc}
\hline Diabetes & Cardiovascular diseases \\
\hline Survival rate of isolated islets $\uparrow$ & Antioxidant capacity $\uparrow$ \\
Glucose tolerance $\uparrow$ & Endothelial function $\uparrow$ \\
Vascular reactivity $\uparrow$ & VSMC invasive activity $\downarrow$ \\
Blood glucose levels $\downarrow$ & VSMC adhesion and migration $\downarrow$ \\
Insulin signaling blockade $\downarrow$ & VSMC proliferation $\downarrow$ \\
Embryonic vasculopathy $\downarrow$ & Adhesion molecule expression $\downarrow$ \\
Pro-inflammatory cytokines $\downarrow$ & Atherosclerotic plaque size $\downarrow$ \\
Lesions of diabetic nephropathy $\downarrow$ & Systolic blood pressure $\downarrow$ \\
\hline
\end{tabular}

VSMC: vascular smooth muscle cell; $\uparrow:$ increase or improve; $\downarrow$ : decrease or attenuate. 
C. Zeng, unpublished data). This strongly supports our hypothesis regarding the beneficial effect of EGCG in macrovascular complications in diabetes.

\section{Cellular and molecular mechanisms of protective effect of EGCG on macrovascular complications}

The underlying mechanisms of EGCG-mediated protective effects on macrovascular complications are not very clear. There are reports showing that EGCG exerts positive actions by attenuation of the apoptotic effect of cytokines, including interleukin $1 \beta$ and interferon- $\gamma$, to human islet cells through the inhibition of nuclear factor$\kappa \mathrm{B}$ activation, which reduces the production of inducible nitric oxide synthase $(24,25)$. Thus, EGCG has a protective effect on islet cells, which may attenuate high glucoseinduced harmful macrovascular effects in diabetes.

Besides the effect on islet cells, EGCG might directly act to prevent/cure macrovascular complications in patients with diabetes. It has been shown that EGCG could prevent vascular reactivity through nitric oxide- and prostaglandin-dependent pathways and via attenuation of aortic lipid peroxidation in diabetic rats (26). It has also been reported that EGCG can inhibit the angiotensin IIstimulated VSMC proliferation via suppression of mitogenactivated protein kinase and nuclear transcription factor

\section{References}

1. Dailey G. Early and intensive therapy for management of hyperglycemia and cardiovascular risk factors in patients with type 2 diabetes. Clin Ther 2011; 33: 665-678, doi: 10.1016/j.clinthera.2011.04.025.

2. Hata J, Arima H, Rothwell PM, Woodward M, Zoungas S, Anderson C, et al. Effects of visit-to-visit variability in systolic blood pressure on macrovascular and microvascular complications in patients with type 2 diabetes mellitus: the ADVANCE trial. Circulation 2013;128: 1325-1334, doi: 10.1161/CIRCULATIONAHA.113.002717.

3. Furukawa S, Sakai T, Niiya T, Miyaoka H, Miyake T, Yamamoto S, et al. Macrovascular Complications and prevalence of urgency incontinence in Japanese patients with type 2 diabetes mellitus: The Dogo Study. Intern Med 2017; 56: 889-893, doi: 10.2169/internalmedicine.56.8063.

4. Silbernagel G, Sourij $H$, Grammer TB, Kleber ME, Hartaigh BO, Winkelmann BR, et al. Isolated post-challenge hyperglycaemia predicts increased cardiovascular mortality. Atherosclerosis 2012; 225: 194-199, doi: 10.1016/j.atherosclerosis. 2012.08.008.

5. Zhang Y, Hu G, Yuan Z, Chen L. Glycosylated hemoglobin in relationship to cardiovascular outcomes and death in patients with type 2 diabetes: a systematic review and metaanalysis. PloS One 2012; 7: e42551, doi: 10.1371/journal. pone.0042551.

6. Guo R, Li W, Liu B, Li S, Zhang B, Xu Y. Resveratrol protects vascular smooth muscle cells against high glucose-induced activator protein-1 signaling pathways (20). Moreover, EGCG is a potent inhibitor of VSMC adhesion by affecting integrin $\beta 1$ expression and binding to extracellular matrix, and is able to inhibit laminin-induced VSMC migration (19). As VSMC proliferation, adhesion and migration are critical events in macrovascular complications, these cellular and molecular mechanisms of EGCG may be effective in the prevention of macrovascular complications in diabetes, which needs to be confirmed in the future.

\section{Conclusion}

Based on previous findings and our preliminary data, we believe that EGCG might be a potential candidate to reduce the occurrence of macrovascular complications in diabetic patients. Confirmation of our hypothesis might provide a novel therapeutic strategy for these patients. Well-designed clinical and laboratory studies could confirm our hypothesis.

\section{Acknowledgments}

The preliminary studies were supported in part by grants from the National Natural Science Foundation of China (\#31430043 and \#81570379) and the National Basic Research Program of China (\#2013CB531104 and \#2012CB517801). oxidative stress and cell proliferation in vitro. Med Sci Monit Basic Res 2014; 20: 82-92, doi: 10.12659/MSMBR.890858.

7. Lin CM, Hou SW, Wang BW, Ong JR, Chang H, Shyu KG. Molecular mechanism of (-)-epigallocatechin-3-gallate on balloon injury-induced neointimal formation and leptin expression. J Agric Food Chem 2014; 62: 1213-1220, doi: $10.1021 / \mathrm{jf} 404479 \mathrm{x}$.

8. Benter IF, Sarkhou F, Al-Khaldi AT, Chandrasekhar B, Attur $S$, Dhaunsi GS, et al. The dual targeting of EGFR and ErbB2 with the inhibitor Lapatinib corrects high glucose-induced apoptosis and vascular dysfunction by opposing multiple diabetes-induced signaling changes. J Drug Target 2015; 23: 506-518, doi: 10.3109/1061186X.2015.1057150.

9. Chen F, Qian LH, Deng B, Liu ZM, Zhao Y, Le YY. Resveratrol protects vascular endothelial cells from high glucose-induced apoptosis through inhibition of NADPH oxidase activation-driven oxidative stress. CNS Neurosci Ther 2013; 19: 675-681, doi: 10.1111/cns.12131.

10. Tsai HY, Huang PH, Lin FY, Chen JS, Lin SJ, Chen JW. Ginkgo biloba extract reduces high-glucose-induced endothelial reactive oxygen species generation and cell adhesion molecule expression by enhancing HO-1 expression via Akt/ eNOS and p38 MAP kinase pathways. Eur J Pharm Sci 2013; 48: 803-811, doi: 10.1016/j.ejps.2013.01.002.

11. Zhao XY, Wang XF, Li L, Zhang L, Shen DL, Li DH, et al. Effects of high glucose on human umbilical vein endothelial cell permeability and myosin light chain phosphorylation. 
Diabetol Metab Syndr 2015; 7: 98, doi: 10.1186/s13098015-0098-0.

12. Brouwers $O$, Niessen PM, Haenen G, Miyata $T$, Brownlee M, Stehouwer $C D$, et al. Hyperglycaemia-induced impairment of endothelium-dependent vasorelaxation in rat mesenteric arteries is mediated by intracellular methylglyoxal levels in a pathway dependent on oxidative stress. Diabetologia 2010; 53: 989-1000, doi: 10.1007/s00125-010-1677-0.

13. Riad A, Westermann D, Van Linthout S, Mohr Z, Uyulmaz S, Becher PM, et al. Enhancement of endothelial nitric oxide synthase production reverses vascular dysfunction and inflammation in the hindlimbs of a rat model of diabetes. Diabetologia 2008; 51: 2325-2332, doi: 10.1007/s00125008-1159-9.

14. Pang J, Zhang Z, Zheng TZ, Bassig BA, Mao C, Liu X, et al. Green tea consumption and risk of cardiovascular and ischemic related diseases: A meta-analysis. Int $\mathrm{J}$ Cardiol 2016; 202: 967-974, doi: 10.1016/j.jcard.2014.12.176.

15. Tenore GC, Daglia M, Ciampaglia R, Novellino E. Exploring the nutraceutical potential of polyphenols from black, green and white tea infusions - an overview. Curr Pharm Biotechnol 2015; 16: 265-271, doi: 10.2174/138920101 6666150118133604.

16. Grassi D, Desideri G, Di Giosia P, De Feo M, Fellini E, Cheli $\mathrm{P}$, et al. Tea, flavonoids, and cardiovascular health: endothelial protection. Am J Clin Nutr 2013; 98: 1660s-1666s, doi: 10.3945/ajcn.113.058313.

17. Hartley L, Flowers N, Holmes J, Clarke A, Stranges S, Hooper L, et al. Green and black tea for the primary prevention of cardiovascular disease. Cochrane Database Syst Rev 2013: Cd009934, doi: 10.1136/jech-2013-203126.109.

18. Cheng XW, Kuzuya M, Nakamura K, Liu Z, Di Q, Hasegawa $J$, et al. Mechanisms of the inhibitory effect of epigallocatechin-3-gallate on cultured human vascular smooth muscle cell invasion. Arterioscler Thromb Vasc Biol 2005; 25: 1864 1870, doi: 10.1161/01.ATV.0000179675.49619.9b.

19. Lo HM, Hung CF, Huang YY, Wu WB. Tea polyphenols inhibit rat vascular smooth muscle cell adhesion and migration on collagen and laminin via interference with cell-ECM interaction. J Biomed Sci 2007; 14: 637-645, doi: 10.1007/ s11373-007-9170-6.

20. Won SM, Park YH, Kim HJ, Park KM, Lee WJ. Catechins inhibit angiotensin Il-induced vascular smooth muscle cell proliferation via mitogen-activated protein kinase pathway. Exp Mol Med 2006; 38: 525-534, doi: 10.1038/emm. 2006.62.

21. Chae YJ, Kim CH, Ha TS, Hescheler J, Ahn HY, Sachinidis A. Epigallocatechin-3-O-gallate inhibits the angiotensin IIinduced adhesion molecule expression in human umbilical vein endothelial cell via inhibition of MAPK pathways. Cell Physiol Biochem 2007; 20: 859-866, doi: 10.1159/ 000110446.

22. Chyu KY, Babbidge SM, Zhao X, Dandillaya R, Rietveld AG, Yano J, et al. Differential effects of green tea-derived catechin on developing versus established atherosclerosis in apolipoprotein E-null mice. Circulation 2004; 109: 24482453, doi: 10.1161/01.CIR.0000128034.70732.C2.

23. Potenza MA, Marasciulo FL, Tarquinio M, Tiravanti E, Colantuono G, Federici A, et al. EGCG, a green tea polyphenol, improves endothelial function and insulin sensitivity, reduces blood pressure, and protects against myocardial I/R injury in SHR. Am J Physiol Endocrinol Metab 2007; 292: E1378-E1387, doi: 10.1152/ajpendo. 00698.2006

24. Fu Z, Zhen W, Yuskavage J, Liu D. Epigallocatechin gallate delays the onset of type 1 diabetes in spontaneous nonobese diabetic mice. Br J Nutr 2011; 105: 1218-1225, doi: 10.1017/S0007114510004824.

25. Song EK, Hur H, Han MK. Epigallocatechin gallate prevents autoimmune diabetes induced by multiple low doses of streptozotocin in mice. Arch Pharm Res 2003; 26: 559-563, doi: 10.1007/BF02976881.

26. Roghani M, Baluchnejadmojarad T. Chronic epigallocatechin-gallate improves aortic reactivity of diabetic rats: underlying mechanisms. Vascul Pharmacol 2009; 51: 8489, doi: 10.1016/j.vph.2009.04.003.

27. Wolfram S, Raederstorff D, Preller M, Wang Y, Teixeira $S R$, Riegger $C$, et al. Epigallocatechin gallate supplementation alleviates diabetes in rodents. J Nutr 2006; 136: 2512-2518.

28. Hara Y, Fujino M, Takeuchi M, Li XK. Green-tea polyphenol (-)-epigallocatechin-3-gallate provides resistance to apoptosis in isolated islets. J Hepatobiliary Pancreat Surg 2007;14: 493-497, doi: 10.1007/s00534-006-1207-0.

29. Yu L, Yu H, Li X, Jin C, Zhao Y, Xu S, et al. P38 MAPK/miR-1 are involved in the protective effect of EGCG in high glucose-induced $\mathrm{Cx} 43$ downregulation in neonatal rat cardiomyocytes. Cell Physiol Biochem 2016; 40: 934-942, doi: 10.1136/jech-2013-203126.109.

30. Lin CL, Lin JK. Epigallocatechin gallate (EGCG) attenuates high glucose-induced insulin signaling blockade in human hepG2 hepatoma cells. Mol Nutr Food Res 2008; 52: 930939, doi: 10.1002/mnfr.200700437.

31. Yang P, Li H. Epigallocatechin-3-gallate ameliorates hyperglycemia-induced embryonic vasculopathy and malformation by inhibition of Foxo3a activation. Am J Obstet Gynecol 2010; 203: 75.e1-6, doi: 10.1016/j.ajog.2010.02.008.

32. Wu CH, Wu CF, Huang HW, Jao YC, Yen GC. Naturally occurring flavonoids attenuate high glucose-induced expression of proinflammatory cytokines in human monocytic THP1 cells. Mol Nutr Food Res 2009; 53: 984-995, doi: 10.1002/ mnfr.200800495.

33. Yamabe N, Yokozawa T, Oya T, Kim M. Therapeutic potential of (-)-epigallocatechin 3-O-gallate on renal damage in diabetic nephropathy model rats. $J$ Pharmacol Exp Ther 2006; 319: 228-236, doi: 10.1124/jpet.106.107029.

34. Chow HH, Cai Y, Hakim IA, Crowell JA, Shahi F, Brooks CA, et al. Pharmacokinetics and safety of green tea polyphenols after multiple-dose administration of epigallocatechin gallate and polyphenon $\mathrm{E}$ in healthy individuals. Clin Cancer Res 2003; 9: 3312-3319.

35. Mineharu $\mathrm{Y}$, Koizumi A, Wada $\mathrm{Y}$, Iso $\mathrm{H}$, Watanabe $\mathrm{Y}$, Date $\mathrm{C}$, et al. Coffee, green tea, black tea and oolong tea consumption and risk of mortality from cardiovascular disease in Japanese men and women. J Epidemiol Community Health 2011; 65: 230-240, doi: 10.1136/jech.2009.097311.

36. Hayat $\mathrm{K}$, Iqbal $\mathrm{H}$, Malik $U$, Bilal U, Mushtaq S. Tea and its consumption: benefits and risks. Crit Rev Food Sci Nutr 2015; 55: 939-954, doi: 10.1080/10408398.2012. 678949.

37. Isbrucker RA, Bausch J, Edwards JA, Wolz E. Safety studies on epigallocatechin gallate (EGCG) preparations. 
Part 1: genotoxicity. Food Chem Toxicol 2006; 44: 626-635, doi: 10.1016/j.fct.2005.07.005.

38. Isbrucker RA, Edwards JA, Wolz E, Davidovich A, Bausch J. Safety studies on epigallocatechin gallate (EGCG) preparations. Part 2: dermal, acute and short-term toxicity studies. Food Chem Toxicol 2006; 44: 636-650, doi: 10.1016/ j.fct.2005.11.003.

39. Isbrucker RA, Edwards JA, Wolz E, Davidovich A, Bausch J. Safety studies on epigallocatechin gallate (EGCG) preparations.
Part 3: teratogenicity and reproductive toxicity studies in rats. Food Chem Toxicol 2006; 44: 651-661, doi: 10.1016/ j.fct.2005.11.002.

40. Yang J, Han Y, Sun H, Chen C, He D, Guo J, et al. (-)-Epigallocatechin gallate suppresses proliferation of vascular smooth muscle cells induced by high glucose by inhibition of PKC and ERK1/2 signalings. J Agric Food Chem 2011; 59: 11483-11490, doi: 10.1021/ jf2024819. 